\title{
\begin{tabular}{l|l} 
MitTraries & DSpace@MIT
\end{tabular}
}

\author{
MIT Open Access Articles
}

Upper bounds to error probability with feedback

The MIT Faculty has made this article openly available. Please share how this access benefits you. Your story matters.

Citation: Nakiboglu, B., and Lizhong Zheng. “Upper bounds to error probability with feedback." Information Theory, 2009. ISIT 2009. IEEE International Symposium on. 2009. 1515-1519. (C) 2009, IEEE

As Published: http://dx.doi.org/10.1109/ISIT.2009.5205849

Publisher: Institute of Electrical and Electronics Engineers

Persistent URL: http://hdl.handle.net/1721.1/60017

Version: Final published version: final published article, as it appeared in a journal, conference proceedings, or other formally published context

Terms of Use: Article is made available in accordance with the publisher's policy and may be subject to US copyright law. Please refer to the publisher's site for terms of use. 


\section{Upper Bounds to Error Probability with Feedback}

\author{
Barış Nakiboğlu \\ Electrical Engineering and Computer Science \\ Massachusetts Institute of Technology \\ Cambridge, MA, 02139 \\ Email: nakib@mit.edu
}

\author{
Lizhong Zheng \\ Electrical Engineering and Computer Science \\ Massachusetts Institute of Technology \\ Cambridge, MA, 02139 \\ Email:lizhong@mit.edu
}

\begin{abstract}
A new technique is proposed for upper bounding the error probability of fixed length block codes with feedback. Error analysis is inspired by Gallager's error analysis for block codes without feedback. Zigangirov-D'yachkov ( $Z-D)$ encoding scheme is analyzed with the technique on binary input channels and $k$ ary symmetric channels. A strict improvement is obtained for $k$-ary symmetric channels.
\end{abstract}

\section{INTRODUCTION}

Shannon showed [8] that capacity of the discrete memoryless channels (DMCs) does not increase with feedback. Later Dobrushin [3] showed that the exponential decay rate of the error probability of fixed length block codes can not exceed sphere packing exponent in symmetric channels. ${ }^{1}$ In other words for the rates above the critical rate, at least for symmetric channels, even the error exponent does not increase with feedback, when we restrict ourselves to the fixed length block codes. Characterizing the improvement in the error exponent for the rates below the critical rate is the pressing open question in this stream of research. ${ }^{2}$

The first work on the error analysis of block codes with feedback was by Berlekamp, [1]. He has obtained a closed form expression of the error exponent at zero rate for binary symmetric channels (BSCs). Later Zigangirov [9] proposed an encoding scheme, for BSCs which reaches sphere packing exponent for all rate larger than a critical rate $R_{Z \text { crit. }} .{ }^{3}$ Furthermore at zero rate Zigangirov's encoding scheme reaches optimal error exponent, which is derived by Berlekamp in [1]. Later D'yachkov [4] proposed a generalization of the encoding scheme of Zigangirov, and obtained a coding theorem for general DMCs. However the optimization problem in his coding theorem, is quite involved and does not allow for

\footnotetext{
${ }^{1}$ After that Haratounian [7] established an upper bound for the error exponent for non-symmetric channels as a generalization of Dobrushin's result, but his upper bound is strictly larger than the sphere packing exponent for many non-symmetric channels.

${ }^{2}$ There are a number of closely related models in which error exponent analysis has been successfully applied, like variable-length block codes, fixed length block codes with errors-and-erasure decoding, block codes on additive white Gaussian noise channels, fixed/variable delay code on DMCs. We are refraining from discussing these variants because understanding those variants will not help the reader much in understanding the work at hand.

${ }^{3}$ Evidently $R_{Z \text { crit }}<R_{\text {crit }}$ where $R_{\text {crit }}$ is the critical rate in the nonfeedback case, i.e. the rate above which random coding exponent is equal to the sphere packing exponent.
}

simplifications that will lead us to conclusions about the error exponents of general DMCs. In [4] after pointing out this fact, D'yachkov focuses on binary input channels and $k$-ary symmetric channels and derives the error exponent expressions for these families of channels.

Our approach will be similar to D'yachkov's in the sense that we will first prove a coding theorem for general DMCs and then focus on particular cases and demonstrate its gains over non-feedback encoding schemes. We will start with introducing the channel model we have at hand and the notation we use. After that we will consider the encoding schemes that use feedback and make an error analysis which is similar to that of Gallager in [5]. Then we will use our results in different cases, to recover the results of Zigangirov [9] and D'yachkov [4] for binary input channels and to improve D'yachkov's results [4] for $k$-ary symmetric channels. ${ }^{4}$

\section{Channel Model and Notation}

We have a discrete memoryless channel with input alphabet $\mathcal{X}=\{1,2, \ldots,|\mathcal{X}|\}$, output alphabet $\mathcal{Y}=\{1,2, \ldots,|\mathcal{Y}|\}$. Channel transition probabilities are given by a $|\mathcal{X}|$-by- $|\mathcal{Y}|$ matrix $W(y \mid x)$. In addition we assume that a noiseless, delay free feedback link exists from the receiver to the transmitter. Thus the transmitter learns the channel output at time $k$ before the transmission of the symbol at time $k+1$.

A length $\mathbf{n}$ block code with feedback for a message set $\mathcal{M}=\left\{1,2, \ldots,\left\lceil e^{\mathbf{n} R}\right\rceil\right\}$ is a feedback encoding scheme together with a decoding rule. The feedback encoding scheme, ${ }^{5}$ $\Psi$, is a mapping from the set of possible output sequences, $y^{j-1} \in \mathcal{Y}^{j-1}$ for $j \in\{1,2, \ldots, \mathbf{n}\}$, to the set of possible input symbol assignment to the messages in the set $\mathcal{M}$

$$
\Psi(\cdot): \bigcup_{j=1}^{\mathrm{n}} \mathcal{Y}^{j-1} \rightarrow \mathcal{X}^{|\mathcal{M}|}
$$

The input letter for the message $m \in \mathcal{M}$ at time $j$ given $y^{j-1} \in \mathcal{Y}^{j-1}$ is the $m^{t h}$ element of $\Psi\left(y^{j-1}\right)$, i.e. $\Psi_{m}\left(y^{j-1}\right)$. Note that when there is no feedback $\Psi\left(y^{j-1}\right)=\Psi(j)$, $\forall y^{j-1} \in \mathcal{Y}^{j-1}$.

\footnotetext{
${ }^{4}$ Indeed same improvement can be obtained within framework of the analysis introduced by Zigangirov and D'yachkov with some fairly minor modifications.

${ }^{5}$ Note that this is the general setting we will focus on a particular mapping
} to establish our results. 
The probability of observing a $y^{i} \in \mathcal{Y}^{i}$ conditioned on message $m \in \mathcal{M}$ is,

$$
\mathbf{P}\left\{y^{i} \mid \theta=m\right\}=\prod_{j=1}^{i} W\left(y_{j} \mid \Psi_{m}\left(y^{j-1}\right)\right)
$$

A decoding rule is a mapping from the set of all length $\mathbf{n}$ output sequences, $\mathcal{Y}^{\mathrm{n}}$, to the message set $\mathcal{M}$.

$$
\Phi(\cdot): \mathcal{Y}^{\mathbf{n}} \rightarrow \mathcal{M}
$$

We use a maximum likelihood (ML) decoder, which decodes the message with the smaller index when there is tie. We assume that messages are equally likely.

\section{ERROR ANALYSIS}

The error probability of the message $m \in \mathcal{M}$ is,

$$
P_{\mathbf{e}, m}=\sum_{y^{\mathbf{n}} \in \mathcal{Y}^{\mathbf{n}}} \mathbf{P}\left\{y^{\mathbf{n}} \mid \theta=m\right\} \mathbb{I}\left\{\Phi\left(y^{\mathbf{n}}\right) \neq m\right\}
$$

where $\mathbb{I}\{\cdot\}$ is the indicator function. Note that for a ML decoder, for any $\rho>0$, and $\eta>0$ we have

$$
\mathbb{I}\left\{\Phi\left(y^{\mathbf{n}}\right) \neq m\right\} \leq\left(\frac{\sum_{k \neq m} \mathbf{P}\left\{y^{\mathbf{n}} \mid \theta=k\right\}^{\eta}}{\mathbf{P}\left\{y^{\mathbf{n}} \mid \theta=m\right\}^{\eta}}\right)^{\rho}
$$

Consequently

$P_{\mathbf{e}, m} \leq \sum_{y^{\mathbf{n}} \in \mathcal{Y}^{\mathbf{n}}} \mathbf{P}\left\{y^{\mathbf{n}} \mid \theta=m\right\}^{1-\rho \eta}\left(\sum_{k \neq m} \mathbf{P}\left\{y^{\mathbf{n}} \mid \theta=k\right\}^{\eta}\right)^{\rho}$

If we introduce the short hand

$\Gamma_{\rho, \eta, j}\left(y^{j}\right)=\sum_{m \in \mathcal{M}} \mathbf{P}\left\{y^{j} \mid \theta=m\right\}^{1-\rho \eta}\left[\sum_{k \neq m} \mathbf{P}\left\{y^{j} \mid \theta=k\right\}^{\eta}\right]^{\rho}$

Since all the messages are equally likely $P_{\mathbf{e}}=\frac{\sum_{m} P_{\mathbf{e}, m}}{|\mathcal{M}|}$. Consequently,

$$
P_{\mathbf{e}} \leq \sum_{y^{\mathbf{n}} \in \mathcal{Y}^{\mathbf{n}}} \frac{\Gamma_{\rho, \eta, \mathbf{n}}\left(y^{\mathbf{n}}\right)}{|\mathcal{M}|}
$$

If $\Gamma_{\rho, \eta, \mathbf{n}-1}\left(y^{\mathbf{n}-1}\right) \neq 0$ we can divide and multiply by $\Gamma_{\rho, \eta, \mathbf{n}-1}\left(y^{\mathbf{n}-1}\right)$,

$$
\begin{aligned}
P_{\mathbf{e}} & \leq \sum_{y^{\mathbf{n}} \in \mathcal{Y}^{\mathbf{n}}} \frac{\Gamma_{\rho, \eta, \mathbf{n}}\left(y^{\mathbf{n}}\right)}{|\mathcal{M}|} \\
& =\sum_{y^{\mathbf{n}} \in \mathcal{Y}^{\mathbf{n}}} \frac{\Gamma_{\rho, \eta, \mathbf{n}-1}\left(y^{\mathbf{n}-1}\right)}{|\mathcal{M}|} \frac{\Gamma_{\rho, \eta, \mathbf{n}}\left(y^{\mathbf{n}}\right)}{\Gamma_{\rho, \eta, \mathbf{n}-1}\left(y^{\mathbf{n}-1}\right)} \\
& =\sum_{y^{\mathbf{n}-1} \in \mathcal{Y}^{\mathbf{n}-1}} \frac{\Gamma_{\rho, \eta, \mathbf{n}-1}\left(y^{\mathbf{n}-1}\right)}{|\mathcal{M}|} \sum_{y_{\mathbf{n}} \in \mathcal{Y}} \frac{\Gamma_{\rho, \eta, \mathbf{n}}\left(y^{\mathbf{n}}\right)}{\Gamma_{\rho, \eta, \mathbf{n}-1}\left(y^{\mathbf{n}-1}\right)}
\end{aligned}
$$

Thus

$$
P_{\mathbf{e}} \leq \sum_{y^{\mathbf{n}-1} \in \mathcal{Y}^{\mathbf{n}-1}} \frac{\Gamma_{\rho, \eta, \mathbf{n}-1}\left(y^{\mathbf{n}-1}\right)}{|\mathcal{M}|} \xi\left(\rho, \eta, y^{\mathbf{n}-1}, \Psi\right)
$$

where $\xi\left(\rho, \eta, y^{\mathbf{n}-1}, \Psi\right)$ is given by,

$$
\xi= \begin{cases}0 & \text { if } \Gamma_{\rho, \eta, \mathbf{n}-1}\left(y^{\mathbf{n}-1}\right)=0 \\ \sum_{y_{\mathbf{n}} \in \mathcal{Y}} \frac{\Gamma_{\rho, \eta, \mathbf{n}}\left(y^{\mathbf{n}}\right)}{\Gamma_{\rho, \eta, \mathbf{n}-1}\left(y^{\mathbf{n}-1}\right)} & \text { if } \Gamma_{\rho, \eta, \mathbf{n}-1}\left(y^{\mathbf{n}-1}\right) \neq 0\end{cases}
$$

Let us define $\alpha(\rho, \eta, \Psi)$ as

$$
\alpha(\rho, \eta, \Psi)=\max _{j \in\{1, \ldots \mathbf{n}\}} \max _{y^{j-1} \in \mathcal{Y}^{j-1}} \xi\left(\rho, \eta, y^{j-1}, \Psi\right)
$$

Then

$$
\begin{aligned}
P_{\mathbf{e}} & \leq \sum_{y^{\mathbf{n}-1} \in \mathcal{Y}_{\mathbf{n}-1}} \frac{\Gamma_{\rho, \boldsymbol{\eta}, \mathbf{n}-1}\left(y^{\mathbf{n}-1}\right)}{|\mathcal{M}|} \alpha(\rho, \eta, \Psi) \\
& \leq \frac{\Gamma_{\rho, \eta, 0}\left(y^{0}\right)}{|\mathcal{M}|} \alpha(\rho, \eta, \Psi)^{\mathbf{n}} \\
& \leq|\mathcal{M}-1|^{\rho} \alpha(\rho, \eta, \Psi)^{\mathbf{n}} \\
& \leq e^{\mathbf{n}(\rho R+\ln \alpha(\rho, \eta, \Psi))}
\end{aligned}
$$

Now we find an encoding scheme, $\Psi$, with small $\alpha(\rho, \eta, \Psi)$. For that we will focus on the encoding schemes that are repetitions of the same 'one step encoding function'.

\section{One STEP EnCOding Functions}

Let $\mathcal{Q}^{|\mathcal{M}|}$ be the set of $|\mathcal{M}|$-dimensional vectors whose entries are all non-negative. Let $\Upsilon$ be a parametric function,

$$
\Upsilon_{\rho, \eta}(\cdot, \cdot): \mathcal{Q}^{|\mathcal{M}|} \times \mathcal{X}^{|\mathcal{M}|} \rightarrow R
$$

If $q$ has at least two non-zero entries then $\Upsilon$ is defined as

$$
\begin{aligned}
& \Upsilon_{\rho, \eta}(q, \chi)=\sum_{y, m} \frac{W\left(y \mid \chi_{m}\right)^{(1-\rho \eta)} q_{m}^{\frac{1-\rho \eta}{\eta}}\left(\sum_{k \neq m} W\left(y \mid \chi_{k}\right)^{\eta} q_{k}\right)^{\rho}}{\sum_{m} q_{m}^{\frac{1-\rho \eta}{\eta}}\left(\sum_{k \neq m} q_{k}\right)^{\rho}} \\
& \text { else } \Upsilon_{\rho, \eta}(q, \chi)=0 .
\end{aligned}
$$

Let us introduce the short hand,

$$
\varphi\left(m \mid y^{j}\right)=\mathbf{P}\left\{y^{j} \mid \theta=m\right\}^{\eta}
$$

then we have

$$
\varphi\left(m \mid y^{j}\right)=W\left(y_{j} \mid \Psi_{m}\left(y^{j-1}\right)\right)^{\eta} \varphi\left(m \mid y^{j-1}\right)
$$

We can write $\xi$ in terms of $\Upsilon$ as follows,

$$
\xi\left(\rho, \eta, y^{j-1}, \Psi\right)=\Upsilon_{\rho, \eta}\left(\varphi\left(\cdot \mid y^{j-1}\right), \Psi\left(y^{j-1}\right)\right)
$$

Note that $\xi\left(\rho, \eta, y^{j-1}, \Psi\right)$ depends on the encoding in first $j-1$ time units only through the $|\mathcal{M}|$ dimensional vector $\varphi\left(\cdot \mid y^{j-1}\right)$. Thus if we can find a $\chi_{q}$ for each $q \in \mathcal{Q}^{|\mathcal{M}|}$ such that $\Upsilon_{\rho, \eta}\left(q, \chi_{q}\right)$ is small, we can use these $\Upsilon_{\rho, \eta}\left(q, \chi_{q}\right)$ to obtain an encoding scheme $\Psi$, with small $\alpha(\rho, \eta, \Psi)$ and consequently a block code with small error probability. These mappings are what we call one step encoding function.

An $|\mathcal{M}|$-dimensional one step encoding function, $\mathbb{X}$, is a mapping from $\mathcal{Q}^{|\mathcal{M}|}$ to the set $\mathcal{X}^{|\mathcal{M}|}$, i.e.,

$$
\mathbb{X}: \mathcal{Q}^{|\mathcal{M}|} \rightarrow \mathcal{X}^{|\mathcal{M}|}
$$

With a slight abuse of notation let us extend the definition of $\alpha(\rho, \eta, \mathbb{X})$ to one step encoding functions as follows,

$$
\alpha(\rho, \eta, \mathbb{X})=\max _{q \in \mathcal{Q}^{|\mathcal{M}|}} \Upsilon_{\rho, \eta}(q, \mathbb{X}(q))
$$


Lemma 1: For any $\rho>0, \eta>0,|\mathcal{M}|=e^{\mathbf{n} R}$ dimensional one step encoding function $\mathbb{X}$, and for any $\mathbf{n} \geq 1$ there exists a block code such that

$$
P_{\mathbf{e}} \leq e^{\mathbf{n}(\ln \alpha(\rho, \eta, \mathbb{X})+\rho R)} .
$$

Proof: Consider the encoding scheme, $\Psi$ such that,

$$
\Psi_{m}\left(y^{j-1}\right)=\mathbb{X}_{m}\left(\varphi\left(\cdot \mid y^{j-1}\right)\right)
$$

As a result of equations (4), (7) and (8) we get,

$$
\alpha(\rho, \eta, \Psi) \leq \alpha(\rho, \eta, \mathbb{X})
$$

Using equation (5) we get the claim of the lemma.

When calculating the achievable error exponents the role of the minimum of $-\ln \alpha(\rho, \eta, \mathbb{X})$ will be very similar to that of $E_{0}(\rho)$ in the case without feedback, [5].

\section{MAIN RESUlts:}

\section{A. Achievability of Random Coding Exponent:}

In this subsection we will, as a sanity check, rederive the achievability of random coding exponent for all DMCs using Lemma 1. Let $\eta=\frac{1}{1+\rho}$. For any $|\mathcal{M}|>1$, at each $q \in$ $\mathcal{Q}$ consider the set of all possible mappings of messages to the input letters, $\mathcal{X}^{|\mathcal{M}|}$, and calculate the expected value of $\Upsilon_{\rho, \eta}(q, \chi)$, where the probability of each $\chi \in \mathcal{X}^{|\mathcal{M}|}$ is simply given by $\prod_{x \in \mathcal{X}} P(x)^{r(\chi, x)}$ where $r(\chi, x)$ is the number of messages assigned to input letter $x \in \mathcal{X}$. Then one can show that, for $\rho \in(0,1]$

$$
\mathbf{E}\left[\Upsilon_{\rho, \frac{1}{1+\rho}}(q, \chi)\right] \leq e^{-E_{0}(\rho, P)} \quad \forall q \in \mathcal{Q}^{|\mathcal{M}|}
$$

where $E_{0}(\rho, P)=-\ln \sum_{y}\left(\sum_{x} W(y \mid x)^{\frac{1}{1+\rho}} P(x)\right)^{1+\rho}$ Thus for each $q \in \mathcal{Q}^{|\mathcal{M}|}$ there exist at least one $\chi_{q} \in \mathcal{X}^{|\mathcal{M}|}$ such that

$$
\Upsilon_{\rho, \frac{1}{1+\rho}}\left(q, \chi_{q}\right) \leq e^{-E_{0}(\rho, P)}
$$

Thus for the one step encoding function $\mathbb{X}(q)=\chi_{q}$ we have

$$
-\ln \alpha\left(\rho, \frac{1}{1+\rho}, \mathbb{X}\right) \geq E_{0}(\rho, P)
$$

Using this together with the lemma 1 we can conclude that; Corollary 1: For any input distribution $P(\cdot)$ on $\mathcal{X}, \rho \in(0,1]$, $R \geq 0$ and $\mathbf{n} \geq 1$ there exists a length $\mathbf{n}$ block code of the form given in equation (10) such that.

$$
P_{\mathbf{e}} \leq e^{\mathbf{n}\left(-E_{0}(\rho, P)+\rho R\right)}
$$

Note that above description is not constructive in the sense that it proves the existence of a one-step-encoding scheme, $\mathbb{X}$ with the desired properties but it does not tell which encoding scheme it is or how to find it. Encoding scheme we will investigate below however does specify an $\mathbb{X}$ with the desired properties.

\section{B. Z-D Encoding Scheme:}

In this subsection we will describe the $Z-D$ encoding scheme and apply Lemma 1 to this encoding scheme on binary input channels and $k$-ary symmetric channels. This encoding scheme was first described by Zigangirov [9] for binary symmetric channels then generalized by D'yachkov [4] to general DMCs. Consider a probability distribution a $P(\cdot)$ on input alphabet $\mathcal{X}$ and a $q \in \mathcal{Q}^{|\mathcal{M}|}$. Without loss of generality we can assume that ${ }^{6} \forall i, j \in \mathcal{M}$, if $i \leq j$ then $q_{i} \geq q_{j}$. Now we can define mapping $\chi$ for a given $q$ and $P$ iteratively as follows:

$$
\begin{aligned}
\gamma_{0}(x) & =0 \quad \forall x \in \mathcal{X} \\
\chi_{j} & =\underset{x \in \operatorname{supp}(P)}{\operatorname{argmin}} \frac{\gamma_{j-1}(x)}{P(x)} \\
\gamma_{j}(x) & =\sum_{1 \leq i \leq j: \chi_{i}=x} q_{i}
\end{aligned}
$$

For assigning $j \in \mathcal{M}$ we first calculate for each input letter, $x \in \mathcal{X}$, the total mass of all of the messages that has already been assigned to $x, \gamma_{j-1}(x)$. Then we divide $\gamma_{j-1}(x)$ 's by the corresponding $P(x)$ values and assign the message $j \in \mathcal{M}$ to the $x \in \mathcal{X}$, for which $P(x)>0$ and $\frac{\gamma_{j-1}(x)}{P(x)}$ is the minimum. If there is a tie we choose the input letter, $x$, with larger $P(x)$. If there is still a tie, we choose the input letter with smaller index.

1) Properties of Z-D Encoding Scheme: A Z-D encoding scheme with $P(\cdot)$, will satisfy,

$$
\zeta_{m}=\frac{q_{\chi_{m}}-q_{m}}{P\left(\chi_{m}\right)} \leq \frac{q_{x}}{P(x)} \quad \forall x \in \mathcal{X} \quad \forall m \in \mathcal{M}
$$

where $q_{x}=\gamma_{|\mathcal{M}|}(x)$. In order to see this, simply consider the last message assigned to each input letter $x \in \mathcal{X}$. They will satisfy this property by construction. Since the messages that are assigned to the same letter prior to the last message have at least the same mass as the last one, they will satisfy the property given in (15) too. Thus for any $q \in \mathcal{Q}^{|\mathcal{M}|}$ and any input distribution $P(x)$, the mapping created by a $Z$ $D$ encoding scheme, satisfies

$$
q_{x}-P(x) \zeta_{m} \geq 0 \quad \forall x \in \mathcal{X} \quad \forall m \in \mathcal{M}
$$

Thus

$$
\frac{\sum_{k \neq m} \mathbb{I}\left\{\chi_{k}=x\right\} q_{k}}{\sum_{k \neq m} q_{k}}=\frac{\zeta_{m} P(x)}{\sum_{k \neq m} q_{k}}+\frac{\sum_{x \neq \chi_{m}} \mathbb{I}\left\{\chi_{k}=x\right\}\left(q_{x}-\zeta_{m} P(x)\right)}{\sum_{k \neq m} q_{k}}
$$

In other words, with $Z-D$ encoding scheme, the mass of the $q$ is distributed over the input letters in such a way that; when we consider all the mass distribution except an $m \in \mathcal{M}$, it is a linear combination of $P(x)$ and $\delta_{x, x^{\prime}}$ 's for $x^{\prime} \neq \chi_{m}$. Using this decomposition of the input distribution together with the convexity of the function $z^{\rho}$ for $\rho \geq 1$ and Jensen's inequality

\footnotetext{
${ }^{6}$ If this is not the case for a $q$, we can rearrange the messages $m \in \mathcal{M}$, according to their $q_{m}$ in decreasing order. If two or more messages have same mass, $q$, we order them with respect to their indices.
} 
we get,

$$
\begin{aligned}
& {\left[\frac{\sum_{x} W(y \mid x)^{\eta} \sum_{k \neq m} \mathbb{I}\left\{\chi_{k}=x\right\} q_{k}}{\sum_{k \neq m} q_{k}}\right]^{\rho}} \\
& \quad=\left[\sum_{x} W(y \mid x)^{\eta}\left(\frac{\zeta_{m} P(x)}{\sum_{k \neq m} q_{k}}+\frac{\sum_{x \neq x_{m}} \mathbb{I}\left\{\chi_{k}=x\right\}\left(q_{x}-\zeta_{m} P(x)\right)}{\sum_{k \neq m} q_{k}}\right)\right]^{\rho} \\
& \quad \leq \frac{\zeta_{m}\left[\sum_{x} W(y \mid x)^{\eta} P(x)\right]^{\rho}}{\sum_{k \neq m} q_{k}}+\sum_{x \neq \chi_{m}} \frac{\left(q_{x}-\zeta_{m} P(x)\right) W(y \mid x)^{\eta \rho}}{\sum_{k \neq m} q_{k}}
\end{aligned}
$$

Let us define $f_{m}$ as

$$
f_{m}=\sum_{y} W\left(y \mid \chi_{m}\right)^{(1-\rho \eta)}\left(\frac{\sum_{k \neq m} W\left(y \mid \chi_{k}\right)^{\eta} q_{k}}{\sum_{k \neq m} q_{k}}\right)^{\rho}
$$

Using equation (17) we get,

$$
\begin{aligned}
f_{m} & \leq \frac{\zeta_{m}}{\sum_{k \neq m} q_{k}} \sum_{y} W\left(y \mid \chi_{m}\right)^{(1-\rho \eta)}\left[\sum_{x} W(y \mid x)^{\eta} P(x)\right]^{\rho} \\
& +\sum_{x \neq \chi_{m}} \frac{\left(q_{x}-P(x) \zeta_{m}\right)}{\sum_{k \neq m} q_{k}} \sum_{y} W\left(y \mid \chi_{m}\right)^{(1-\rho \eta)} W(y \mid x)^{\rho \eta} \\
& \leq \frac{\zeta_{m}}{\sum_{k \neq m} q_{k}} e^{\beta_{\chi_{m}}(P, \rho, \eta)}+\left(1-\frac{\zeta_{m}}{\sum_{k \neq m} q_{k}}\right) e^{\mu_{\chi m}(\rho \eta)} \\
& \leq e^{\max \left\{\beta_{\chi_{m}}(P, \rho, \eta), \mu_{\chi m}(\rho \eta)\right\}}
\end{aligned}
$$

where $\forall i \in \mathcal{X}, \beta_{i}(P, \rho, \eta)$ and $\mu_{i}(\rho \eta)$ are defined as,

$$
\begin{aligned}
\beta_{i}(P, \rho, \eta) & =\ln \sum_{y} W(y \mid i)^{(1-\rho \eta)}\left(\sum_{x} P(x) W(y \mid x)^{\eta}\right)^{\rho} \\
\mu_{i}(\rho \eta) & =\max _{x \neq i, x \in \operatorname{supp}(P)} \ln \sum_{y} W(y \mid i)^{(1-\rho \eta)} W(y \mid x)^{\rho \eta}
\end{aligned}
$$

Consequently for $\rho \geq 1, \eta \geq 0$,

$$
\begin{aligned}
& \Upsilon_{\rho, \eta}\left(q, \chi_{P}\right)=\frac{\sum_{y, m} W\left(y \mid \chi_{m}\right)^{(1-\rho \eta)} q_{m}^{\frac{1-\rho \eta}{\eta}}\left(\sum_{k \neq m} W\left(y \mid \chi_{k}\right)^{\eta} q_{k}\right)^{\rho}}{\sum_{m} q_{m}^{\frac{1-\rho \eta}{\eta}}\left(\sum_{k \neq m} q_{k}\right)^{\rho}} \\
& =\frac{\sum_{m} q_{m}^{\frac{1-\rho \eta}{\eta}}\left(\sum_{k \neq m} q_{k}\right)^{\rho} f_{m}}{\sum_{m} q_{m}^{\frac{1-\rho \eta}{\eta}}\left(\sum_{k \neq m} q_{k}\right)^{\rho}} \\
& \leq e^{\max _{x \in \operatorname{supp} P(\cdot)} \max \left\{\beta_{x}(P, \rho, \eta), \mu_{x}(\rho \eta)\right\}}
\end{aligned}
$$

Thus for $\rho \geq 1$ for all input distributions $P$ and for all $\eta>0$,

$$
\ln \alpha\left(\rho, \eta, \mathbb{X}_{P}\right) \leq \max _{x \in \operatorname{supp} P(\cdot)} \max \left\{\beta_{x}(P, \rho, \eta), \mu_{x}(\rho \eta)\right\}
$$

For certain channels the property given in equation (19) together with Lemma 1 implies that sphere packing exponent is achievable on an interval of the form $\left[R_{\text {Dcrit }}, R_{\text {crit }}\right]$.

Corollary 2: If for a DMC,

$$
\max _{x \in \mathcal{X}} \mu_{x}\left(\frac{\rho}{1+\rho}\right) \leq-E_{0}(\rho)
$$

on an interval of the form $\rho \in\left[1, \rho_{D c}\right]$. Then

$$
\ln \alpha\left(\rho, \frac{1}{1+\rho}, \mathbb{X}\right)=-E_{0}(\rho) \quad \forall \rho \in\left[1, \rho_{D c}\right]
$$

Proof: In order to see this, first recall that for the $P(\cdot)$ that maximizes $E_{0}(\rho, P)$, satisfies, [6, page 144, Theorem 5.6.5],

$$
E_{0}\left(\rho, P^{*}\right)=\beta_{x}\left(P^{*}, \frac{1}{1+\rho}, \rho\right) \quad \forall x \in \operatorname{supp}\left(P^{*}\right)
$$

ISIT 2009, Seoul, Korea, June 28 - July 3, 2009

Now the statement simply follows the equation (19)

Recall that sphere packing exponent is given by

$$
E_{s p}(R)=\max _{\rho \geq 0} E_{0}(\rho)-\rho R
$$

Thus for each $R$ there is a corresponding $\rho_{R}$, which is the maximizer of the the expression above. As a result of Lemma1 and Corollary 2 for the rates with $\rho_{R} \in\left[1, \rho_{D c}\right]$ sphere packing exponent will be achievable as error exponent for the channels satisfying condition given in equation (20). Two families of channels that satisfy the condition (20) are Binary input channels and $k$-ary symmetric channels.

2) Binary Input Channel: The binary input channel case has already been addressed by D'yachkov. We simply rederive his results here. DMCs for which $|\mathcal{X}|=2$ are called binary input channels.

For $\rho \geq 1, \eta \geq 0$, using equation (19) we get,

$$
\alpha(\rho, \eta, \mathbb{X}) \leq e^{\max \left\{\beta_{0}(P, \eta, \rho), \beta_{1}(P, \eta, \rho), \mu_{0}(\rho \eta), \mu_{1}(\rho \eta)\right\}}
$$

For the rates for which $\rho_{R} \in\left[1, \rho_{D c}\right]$ this will lead to sphere packing exponent. For the rates for which $\rho_{R}>\rho_{D c}$ we will simply minimize the expression in equation (21) over $P, \eta$ and $\rho$ to find the best possible error exponent achievable with this scheme. For the rates such that $\rho_{R} \in(0,1)$ using the definition of $f_{m}$ given in equation (18) and certain monotonicity argument one can also show that sphere packing exponent is achievable. We do not present those arguments in the interest of space.

3) $K$-ary Symmetric Channel: Let us consider $k$-ary symmetric channel with $0<\epsilon<\frac{K-1}{K}$, i.e.

$$
w(i \mid j)= \begin{cases}1-\epsilon & i=j \\ \frac{\epsilon}{K-1} & i \neq j\end{cases}
$$

Note that for any $\rho>0, \eta>0$ and $x$ we have

$$
\begin{aligned}
\mu(\rho \eta)= & \mu_{x}(\rho \eta) \\
=\ln \left[(1-\epsilon)\left(\frac{\epsilon}{(K-1)(1-\epsilon)}\right)^{\rho \eta}\right. & \\
& \left.\quad+\frac{\epsilon}{K-1}\left(\frac{(K-1)(1-\epsilon)}{\epsilon}\right)^{\rho \eta}+\frac{\epsilon(K-2)}{K-1}\right]
\end{aligned}
$$

Furthermore note that for any $\rho>0, \eta>0, x \in \mathcal{X}$ and for $P(x)=1 / K$

$$
\begin{aligned}
\beta(\rho, \eta)= & \beta_{x}(P, \rho, \eta) \\
=\ln \left[(1-\epsilon)^{1-\rho \eta}+(K\right. & \left.-1)\left(\frac{\epsilon}{K-1}\right)^{1-\rho \eta}\right] \\
& +\rho \ln \left[\frac{(1-\epsilon)^{\eta}}{K}+\frac{K-1}{K}\left(\frac{\epsilon}{K-1}\right)^{\eta}\right]
\end{aligned}
$$

Thus as a result of equation (19), for $\rho \geq 1$

$$
\ln \alpha(\rho, \eta, \mathbb{X}) \leq \max \{\beta(\rho, \eta), \mu(\rho \eta)\}
$$

For $K=2$ case these expressions are equivalent to those of Zigangirov in [9], which were specifically derived for BSCs. For $K \geq 3$ case these expressions result in a strict 


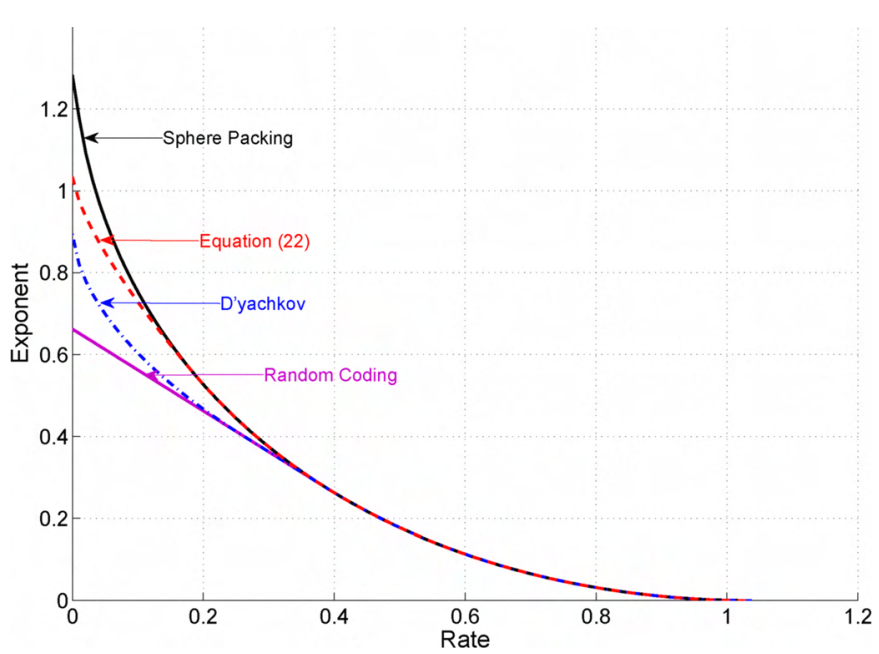

Fig. 1. Spherepacking exponent, error exponent obtained using the equation (22), D'yachkov's exponent expression in [4] and random coding exponent are plotted for a k-ary symmetric channel with $k=4$ and $\epsilon=.3 / 4$.

improvement over [4]. Equivalent of equation (22), in [4] has $\beta_{D}(\rho, \eta)$ instead of $\beta(\rho, \eta)$ where,

$$
\begin{aligned}
\beta_{D}(\rho, \eta)=\ln & {\left[(1-\epsilon)^{1-\rho \eta}\left(\frac{(1-\epsilon)^{\eta}}{K}+\frac{K-1}{K}\left(\frac{\epsilon}{K-1}\right)^{\eta}\right)^{\rho}\right.} \\
& \left.+(K-1)\left(\frac{\epsilon}{K-1}\right)^{1-\rho \eta}\left(\frac{(1-\epsilon)^{\eta \rho}}{K}+\frac{K-1}{K}\left(\frac{\epsilon}{K-1}\right)^{\eta \rho}\right)\right]
\end{aligned}
$$

Note that

$$
\begin{aligned}
\frac{e^{\beta(\rho, \eta)}-e^{\beta}(\rho, \eta)}{(K-1)^{\rho \eta}(1-\epsilon)^{\rho \eta}}= & \left(\frac{(1-\epsilon)^{\eta}}{K}+\frac{(K-1)\left(\frac{\epsilon}{K-1}\right)^{\eta}}{K}\right)^{\rho} \\
& -\left(\frac{(1-\epsilon)^{\rho \eta}}{K}+\frac{(K-1)\left(\frac{\epsilon}{K-1}\right)^{\rho \eta}}{K}\right)
\end{aligned}
$$

For $\rho>1$ as a result of Jensen's inequality $\mathbf{E}[z]^{\rho}<\mathbf{E}\left[z^{\rho}\right]$. Thus $\beta(\rho, \eta)<\beta_{D}(\rho, \eta)$ for all $\rho>1$ and $\eta>0$. Consequently equation (22) leads to $\alpha(\rho, \eta, \mathbb{X})$ 's that are strictly smaller those in [4] for all $\rho>1$. Using Lemma 1 one can convert this improvement into tighter bounds on error exponent. Figure 1 shows the resulting error exponents for a particular channel. ${ }^{7}$

4) Remarks on Z-D Encoding Scheme: The two example channels we have considered do not reveal the main weakness of the $Z-D$ encoding scheme. Consider the following four-byfour symmetric channel $W(y \mid x)$,

$$
W(y \mid x)=\left[\begin{array}{cccc}
1-\epsilon & \epsilon & 0 & 0 \\
0 & 1-\epsilon & \epsilon & 0 \\
0 & 0 & 1-\epsilon & \epsilon \\
\epsilon & 0 & 0 & 1-\epsilon
\end{array}\right]
$$

The error exponent of this channel is equal to sphere packing exponent at all rates. In order to see why recall, [6, page 538, exercise 5.20], $\forall R>0$ there is a fixed list size $L_{R}$ such that for any $\mathbf{n} \geq 1$ there is a length $\mathbf{n}$ block code with with $\left\lfloor e^{\mathbf{n} R}\right\rfloor$ messages and decoding list size $L_{R}$ with error probability $P_{\mathbf{e}} \leq e^{-E_{s p}(R) \mathbf{n}}$. Using $\log _{2} L_{R}$ extra channel

\footnotetext{
${ }^{7}$ Indeed D'yachkov's expression for $k$-ary symmetric channel is strictly worse than that of Zigangirov for $k=2$ case too.
}

uses transmitter can specify the correct message among the decoded list without any errors, if the correct message is in the list. Since this extra time will become negligible as $\mathbf{n}$ increases we can conclude that sphere packing exponent is achievable for this channel, when there is feedback.

However when we consider the $Z-D$ encoding scheme we can not reach the same conclusion. Clearly we should choose the $P(x)$ to be the uniform distribution. Consider for example the $q$, such that $q=\left[\begin{array}{lllll}0.5 & 0.5 & 0 & 0 & \ldots\end{array}\right]$. Any smart encoding scheme would assign the first two messages into input letters that are not consecutive. But $Z-D$ encoding scheme will not necessarily do so. Considering similar $q$ 's for low enough $\rho$ 's one can show that $Z-D$ encoding scheme leads to an $\alpha\left(\rho, \frac{1}{1+\rho}\right)$ such that $\ln \alpha\left(\rho, \frac{1}{1+\rho}\right)>-E_{0}(\rho)$. As a result this will imply that $Z-D$ encoding scheme will have an error exponent strictly less than the sphere pacing exponent.

On the other hand for all such $q$ 's with this kind of anomalies we observed, we were able to find a modification of Z-D encoding scheme, which employs a smarter encoding scheme for the first $|\mathcal{X}|$ messages and performs optimally. However we have yet to find a general modification on $Z-D$ encoding scheme that works for all $q \in \mathcal{Q}$.

\section{CONCLUSIONS}

In addition to the improvement in $k$-ary symteric channel, maybe more importantly, simplicity of the analysis gives us a better understanding of the $Z-D$ encoding scheme in general DMCs. ${ }^{8}$

\section{ACKNOWLEDGEMENT}

Author would like to thank Anand Sarwate and reviewers for their suggestions and corrections on the manuscript.

\section{REFERENCES}

[1] E. R. Berlekamp. Block Coding with Noiseless Feedback. Ph.D. thesis, Massachusetts Institute of Technology, Department of Electrical Engineering, 1964.

[2] M. V. Burnashev. On the reliability function of a binary symmetrical channel with feedback. Problemy Perdachi Informatsii, 24, No. 1:3-10, 1988.

[3] R. L. Dobrushin. An asymptotic bound for the probability error of information transmission through a channel without memory using the feedback. Problemy Kibernetiki, vol 8:161-168, 1962.

[4] A. G. D'yachkov. Upper bounds on the error probability for discrete memoryless channels with feedback. Problemy Perdachi Informatsii, Vol 11, No. 4:13-28, 1975.

[5] R. G. Gallager. A simple derivation of the coding theorem and some applications. Information Theory, IEEE Transactions on, 11, No. 1:3$18,1965$.

[6] R. G. Gallager. Information Theory and Reliable Communication. John Wiley \& Sons, Inc., New York, NY, USA, 1968.

[7] E. A. Haroutunian. A lower bound of the probability of error for channels with feedback. Problemy Peredachi Informatsii, vol 13:36-44, 1977.

[8] C. Shannon. The zero error capacity of a noisy channel. IEEE Transactions on Information Theory, Vol. 2, Iss 3:8-19, 1956.

[9] K. Sh. Zigangirov. Upper bounds for the error probability for channels with feedback. Problemy Perdachi Informatsii, Vol 6, No. 2:87-92, 1970.

${ }^{8}$ On a separate note Burnashev [2] considered the binary symmetric channels and showed that for all the rates between 0 and $R_{Z \text { crit }}$ one can reach and an error exponent strictly higher than the ones given in [9]. A similar modification is possible within our frame work too. 